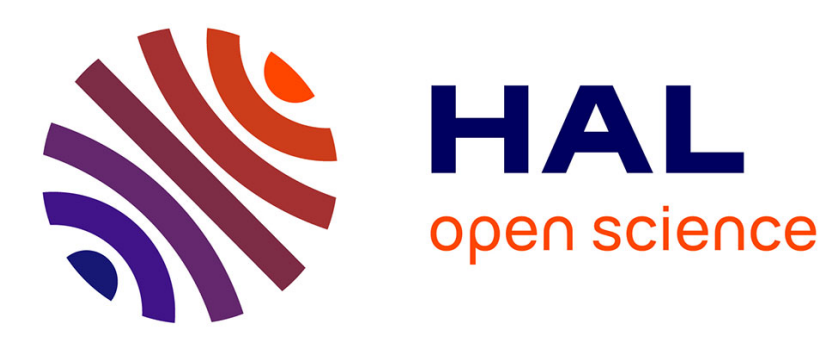

\title{
Quality Assessment of Fingerprints with Minutiae Delaunay Triangulation
}

Z Yao, Jean-Marie Le Bars, Christophe Charrier, Christophe Rosenberger

\section{To cite this version:}

Z Yao, Jean-Marie Le Bars, Christophe Charrier, Christophe Rosenberger. Quality Assessment of Fingerprints with Minutiae Delaunay Triangulation. International Conference on Information Systems Security and Privacy, Feb 2015, Angers, France. hal-01096174

\section{HAL Id: hal-01096174 https://hal.science/hal-01096174}

Submitted on 16 Dec 2014

HAL is a multi-disciplinary open access archive for the deposit and dissemination of scientific research documents, whether they are published or not. The documents may come from teaching and research institutions in France or abroad, or from public or private research centers.
L'archive ouverte pluridisciplinaire HAL, est destinée au dépôt et à la diffusion de documents scientifiques de niveau recherche, publiés ou non, émanant des établissements d'enseignement et de recherche français ou étrangers, des laboratoires publics ou privés. 


\title{
Quality Assessment of Fingerprints with Minutiae Delaunay Triangulation
}

\author{
Z. Yao, J. Le bars, C. Charrier and C. Rosenberger \\ Universite de Caen Basse Normandie; ENSICAEN; UMR 6072 GREYC, Caen, France. \\ \{jean-marie.lebars; christophe.charrier\}@unicaen.fr \{zhigang.yao; christophe.rosenberger $\} @ e n s i c a e n . f r$
}

\begin{abstract}
Keywords: Fingerprint, minutiae template, quality assessment, evaluation.
Abstract: $\quad$ This article proposes a new quality assessment method of fingerprint, represented by only a set of minutiae points. The proposed quality metric is modeled with the convex-hull and Delaunay triangulation of the minutiae points. The validity of this quality metric is verified on several Fingerprint Verification Competition (FVC) databases by referring to an image-based metric from the state of the art (considered as the reference). The experiments of the utility-based evaluation approach demonstrate that the proposed quality metric is able to generate a desired result. We reveal the possibility of assessing fingerprint quality when only the minutiae template is available.
\end{abstract}

\section{INTRODUCTION}

The purpose of fingerprint quality assessment is to improve or guarantee the performance of a biometric system (Grother and Tabassi, 2007) by forbidding bad quality fingerprint samples, especially during the enrollment session. Existing studies of this issue mostly define the quality in terms of the clarity of ridge-valley pattern, contrast between foreground and background, smoothness of orientation and etc (Ratha and Bolle, 1999; Bolle et al., 1999; Chen et al., 2004). No matter how many aspects are considered by a fingerprint quality assessment approach, it is all about features employed for generating the quality metric. Prior studies in estimating fingerprint quality had proposed several features (Alonso-Fernandez et al., 2007), among which some are carried out by using an unique feature, while some others combine multiple features together. For instance, Chen et al. proposed the Fourier spectrum-based quality metric with the Butterworth function, Zhao el al. (Zhao et al., 2010) concluded that the orientation certainty level (OCL) (Lim et al., 2002) also performs well for high resolution fingerprint image and Tabassi et al. (Tabassi et al., 2004) proposed NFIQ with multiple features including minutiae quality. To standardize the quality metric of biometric sample, the quality has been defined as a utility property (Staff, 2009), i.e. sample quality reflects its impact on the performance of the system. However, system performance fully relies on the matching approach such as minutiae-based system which is employed most in actual deployments. In this case, it is reasonable to consider qualifying fingerprint with only minutiae information, particularly when using for embedded applications.

There are very few quality assessment approaches that take into account minutiae information, such as NFIQ. Moreover, none of the state-of-the-art approaches qualifies a fingerprint from the minutiae template alone. The main contribution of the proposed study is a black box algorithm that computes quality score from a minutiae template associated to the fingerprint. In another word, it could be viewed as a quality metric for assessing the quality of a minutiae template. The benefit of having this type of metric is related to embedded biometric systems in smart cards or smart objects where only the minutiae template is available due to computational and storage constraints.

This paper is organized as follows. Section 2 presents the proposed quality metric. Details of the experimental results are given in section 3 . Section 4 concludes the paper and discusses the perspectives.

\section{METRIC DEFINITION}

Fingerprint matching approaches, according to the literature (Maltoni et al., 2009), are broadly classified 
as minutiae-based, correlation-based or image-based, among which minutiae-based is the most widely studied solution. A minutiae template generally provides three kinds of information: 1) minutia location, 2) the orientation and 3 ) the type of minutia point (ridge ending and bifurcation), see figure 1.

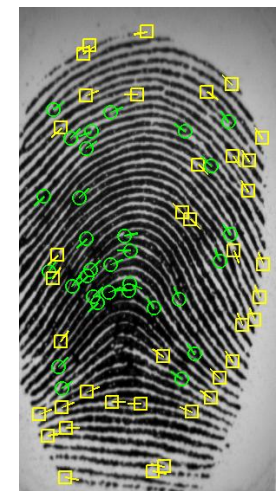

Figure 1: A fingerprint and its minutiae template.

These features are sufficient to reconstruct a synthetic fingerprint from a given template (Feng and Jain, 2011) as the orientation field can be estimated. Another kind of information is the amount of detected minutiae points, which has been used as one factor (Tabassi et al., 2004) for quality assessment of fingerprint samples. However, this information is far from being enough for generating an effective quality metric. In order to do so, the minutiae template is related with the foreground of fingerprint because the area of minutiae indicates an available and useful region for the so-called 'extractability' of features. In this case, this paper proposed to estimate fingerprint quality by modeling the associated minutiae template with the convex-hull and Delaunay triangulation (Cf. figure 2).
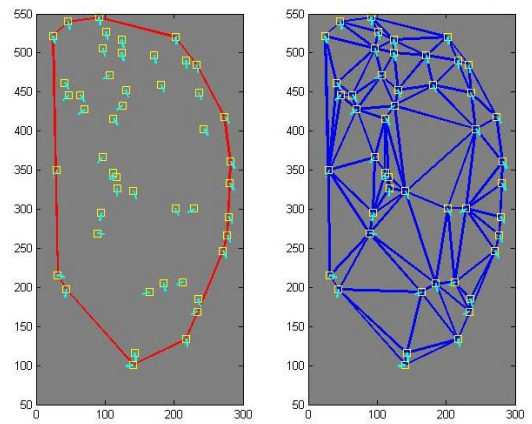

Figure 2: A convex hull (left) and Delaunay Triangulation.

The 2D convex hull (Andrew, 1979) $C_{i}=\left\{\left(x_{j}, y_{j}\right) \mid j=1, \cdots, N_{i}\right\} \quad$ (where $N_{i}$ is the minutiae number of the $i^{\text {th }}$ fingerprint) is defined as the smallest convex set that contains all the points of a given set of minutiae $F_{i}$ in this case (Cf. figure 2). The Delaunay Triangulation of a set of points $P$ in 2D plane is defined as a triangulation DT(P) (Delaunay, 1934) that none of the points of the given set are inside the circumcircle of any triangle of DT(P), as illustrated in figure 2.

With the respective properties of these two geometric structures, an area of the smallest informative region of the fingerprint and a set of areas and perimeters of triangles composed by each 3 minutiae points are obtained. By doing so, one unavoidable problem is observed that almost all the templates of bad quality fingerprints contain both correctly detected minutiae point and spurious points. Based on this observation, we proposed a quality metric as described below.

Let a minutiae template $F_{i}$ of a fingerprint containing a set of detected minutiae points represented by $m_{j}=\left(x_{j}, y_{j}, \theta_{j}\right)$, where $\left(x_{j}, y_{j}\right)$ is the location and $\theta_{j}$ is the orientation of the $j^{\text {th }}$ minutia point; A convex hull denoted by $C_{i}$ and a set of triangles $\left(T_{k}\right)$ formed by Delaunay triangulation, formulated as $D T\left(F_{i}\right)=\left\{T_{k} \mid k=1,2, \cdots, L_{i}\right\}$ where $L_{i}\left(<2 N_{i}\right)$ is the number of triangles can be constructed from $F_{i}$. Correspondingly, the convex hull encloses all the minutiae of $F_{i}$ with the smallest area represented by $A_{i}$, and a set of areas $S_{k}$ and perimeters $P_{k}$ of $D T\left(F_{i}\right)$ are respectively obtained.

In the experiments, we observed that bad quality samples generate tiny and extremely narrow triangles (considered as unreasonable) due to spurious minutiae points. The quantitative values of their area and perimeter are (visually) not proportional to each other, as observed in Figure 3.

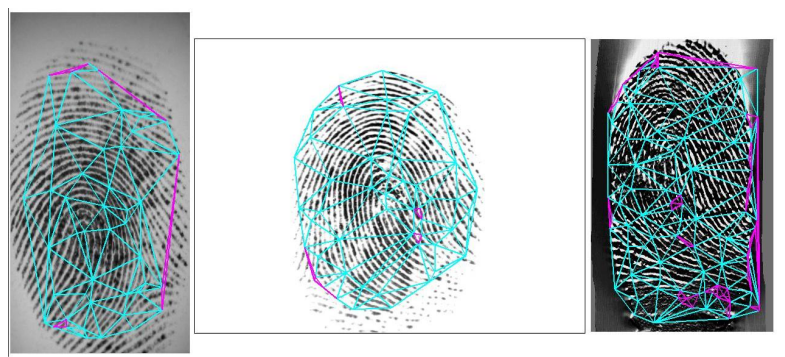

Figure 3: Example of minutiae Delaunay triangulations of 3 different FVC images. NFIQ values are 2, 2 and 1, respectively.

In figure 3, triangles drew by the pink color indicates the unreasonable minutiae structures. Based on these observations, the quality metric (abbreviated as 
MQF afterwards) is calculated with several steps, as described by algorithm 1 . In the computation of the proposed quality metric, three thresholds are set for triangle perimeter, triangle area and the ratio between the perimeter and area of the triangle, which are represented by $Y_{p}, Y_{a}$ and $Y_{r}$, respectively. The details for choosing the most appropriate parameters are discussed in section 3 . The quality value $q$ is dependent on the size of the minutiae region so that it is normalized into the range of $[0,100]$ on each database.

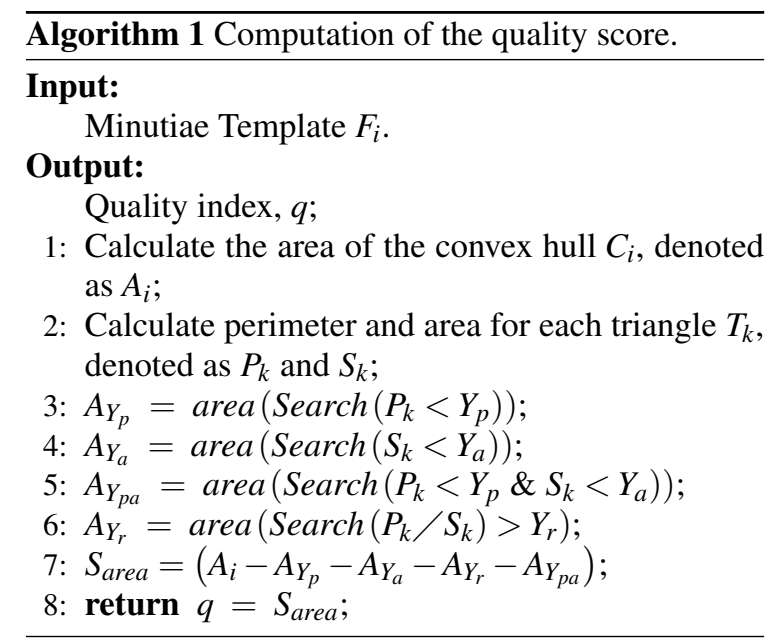

Obviously, this algorithm relates the minutiae template with the area of a region for matching operations as it has just been mentioned before. However, this factor is not sufficient so that we consider to remove a part of potentially useless area from the informative region due to the lack of image information. Delaunay triangulation gives a relatively ideal solution to this problem thanks to the spurious minutiae of bad quality images. For example, as it is depicted in figure 3, some unreasonable triangles formed by spurious minutiae on the border enable us to remove the corresponding area. Another case is the tiny triangle which is mostly happened to the area where the quality of ridge-valley pattern is relatively bad. The study also noted that some fingerprint images have several genuine minutiae clustering in a very small area. This case is not specially considered in the proposed algorithm just because of the limitation of the template. The area of each triangle is obtained by using Heron's formula (Aufmann et al., 2007).

\section{EXPERIMENTAL RESULTS}

In order to validate the MQF, we adopt several approaches to estimate the performance of the qual- ity metric: 1) the Pearson correlation coefficients between the MQF and several others (from the state-ofthe-art) are calculated and 2) the MQF is evaluated by using an utility-based evaluation approach (Yao et al., 2014). The experiment is performed with a laptop driving by an Intel Celeron dual-core CPU of $1.73 \mathrm{GHz}$. The experimental protocol is presented at first.

\subsection{Protocol et Database}

In this study, several FVC databases with different resolutions (Maio et al., 2004) have been used for experiments: FVC2000DB2A, FVC2002DB2A, FVC2004DB1A, FVC2004DB2A and FVC2004DB3A. The first database is established by a low capacitive sensor and the last one relies on a thermal sweeping sensor, while others are established by several optical sensors, see table 1 for details.

Table 1: Details of databases.

\begin{tabular}{|l|c|c|c|}
\hline DB & Sensor Type & Resolution & Image Dim \\
\hline 00DB2A & Capacitive & 500dpi & $256 \times 364$ \\
\hline 02DB2A & Optical & 569dpi & $296 \times 560$ \\
\hline 04DB1A & Optical & 500dpi & $480 \times 640$ \\
\hline 04DB2A & Optical & 500dpi & $328 \times 364$ \\
\hline 04DB3A & Thermal & 512dpi & $300 \times 480$ \\
\hline
\end{tabular}

Each of these databases involves 100 fingerprints, and 8 samples for each fingerprint. In this case, the matching scores involved in the experiment have been calculated by using the NBIS tool (Watson et al., 2007), Bozorth3. The intra-class scores contain $7 \times 100=700$ genuine scores, and the interclass scores consist of $7 \times 99 \times 100=69300$ impostor scores for the whole database. Minutiae templates used in the experiment were also extracted by using the NBIS tool, MINDTCT. This software generates a quadruple representation of minutia point, $m_{i}=\{x, y, o, q\}$, where $(x, y)$ is the location of minutia point, $o$ indicates orientation and $q$ is a quality score of minutia point. In the experiment, the location has been used only for calculating the proposed quality metric.

\subsection{Parameter Settings}

Fernandez et al. (Alonso-Fernandez et al., 2007) and Olsen (Olsen et al., 2012) respectively calculated Pearson and Spearman correlation coefficients between different quality metrics to observe their behavior. Similarly, we investigate the behavior of the proposed quality metric through the Pearson correlation coefficients, by which the parameters are 
appropriately selected as well.

The three thresholds $\left(Y_{p}, Y_{a}\right.$ and $\left.Y_{r}\right)$ are all empirical values observed in the experiments and they are all dependent on the resolution of the image which is supposed to be over $500 \mathrm{dpi}$ in this study. With the experiment, we noted that the smallest area of the triangles of each template is generally less than 70 (considering a histogram of 100 bins). This value is only a scalar without considering the unit of the measurement. In this case, we firstly preferred to choose a value of the area over 70. In addition, it is easy to observe that a threshold larger than 80 may loose the significance of this parameter. The variation of the correlation values (larger than 0.3 ) demonstrates this problem, see figure 4.
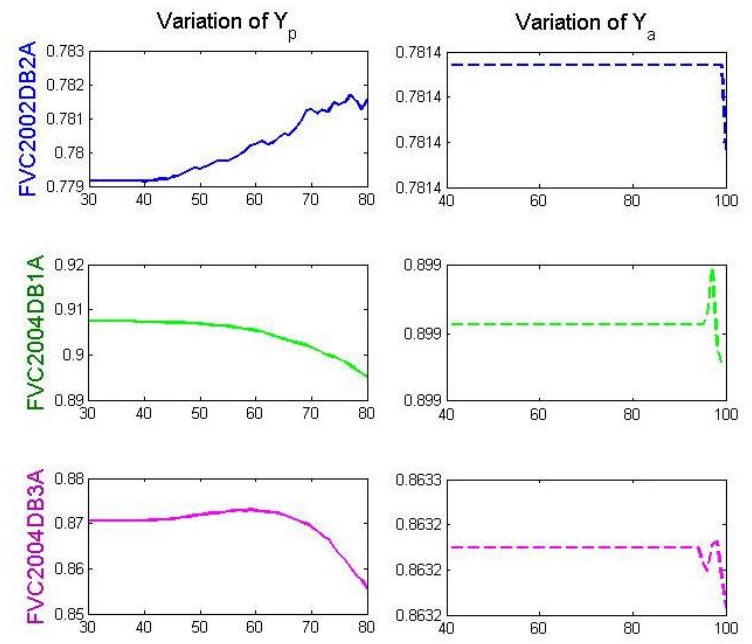

Figure 4: The variation of correlation between the proposed metric and OCL as the parameter changes.

Likewise, the smallest perimeter value of the triangles of each template is mostly within the interval $[10,80]$, and generally lies around 35 (according to the histogram). In this case, we further consider the relation between the area and the perimeter of those triangles that seemed abnormal. Without considering the unit of the two measurements (one is 1-D and another is 2-D), we observed that the value of the perimeter is generally smaller than the area. In this case, inspiring by the Heron's formula, we choose a threshold to represent the ratio between these two measurements. We observed that the triangle is extremely narrow if the ratio between a perimeter and an area is close to 1 . Besides, similarly, it is also not necessary to consider larger values for the perimeter. We first choose a reference quality metric among all the others. The correlation of OCL doesn't vary so much on variant databases. Therefore, we simply choose the OCL in the experiment. Figure 4 provides only the graphical result of the variation of correlation value between OCL and the quality metric obtained with a series values of two parameters. In the experiments, the thresholds of the area and perimeter vary in the range of $[40,100]$ and $[30,80]$ with an interval of 1 , respectively.

According to figure 4, we also observed that the variation of $Y_{p}$ leads to more impacts on the correlation coefficient than $Y_{a}$ when other parameters had been set approximately. In order to achieve a generality of the proposed quality metric, the values of $Y_{p}, Y_{a}$ and $Y_{r}$ in this study are 75, 70 and 0.8, respectively. At last, the behavior of these parameters were estimated by using performance validation approach, and the variation of the performance measurement (EER) obtained in a small interval of each of them tends to be stagnant. With this empirical analysis, the parameters are set as what had just been mentioned.

\subsection{Feature Analysis}

To validate the MQF, we calculate the correlation coefficients between several quality metrics we have studied including the MQF. In addition to the two orientation-based indexes, OCL and the orientation flow (OF) (Lim et al., 2002), we also employ a wavelet domain feature carried out via the Pet Hat's continuous wavelet (PHCWT) (Nanni and Lumini, 2007). In the literature, it is said that the Pet Hat wavelet is sensitive to the sharp variations of features such as fingerprint ridges. Nevertheless, we also use a pixel-based quality metric which is the standard deviation (STD) of fingerprint local block indicating pixel information of the image (Lee et al., 2005). The NFIQ is also involved in this section. Table 2 presents the correlation results of the trial quality metrics.

Table 2: Inter-class Pearson correlation coefficients. FVC2002DB2A

\begin{tabular}{cccccc}
\hline OCL & OF & PHCWT & STD & MQF & NFIQ \\
\hline 1 & 0.013 & 0.932 & 0.892 & 0.781 & -0.503 \\
0.013 & 1 & 0.092 & 0.122 & 0.070 & 0.061 \\
0.932 & 0.092 & 1 & 0.954 & 0.788 & -0.474 \\
0.892 & 0.122 & 0.954 & 1 & 0.678 & -0.374 \\
0.781 & 0.070 & 0.788 & 0.678 & 1 & -0.422 \\
-0.503 & 0.061 & -0.474 & -0.374 & -0.422 & 1 \\
\hline
\end{tabular}

Table 2 provides only an inter-class correlation result of the employed quality metrics obtained from FVC2002DB2A. According to the coefficient values indicated with the highlighted cells, one can observe that MQF demonstrates the correlated behavior with the others except the OF. The correlation coefficients 
between the proposed metric and others calculated from the remaining databases are given in table 3

Table 3: Pearson correlation coefficients between MQF and others.

\begin{tabular}{cccccc}
\hline MQF of & OCL & OF & PHCWT & STD & NFIQ \\
\hline 00DB2 & 0.409 & -0.131 & 0.291 & 0.301 & -0.081 \\
04DB1 & 0.899 & 0.253 & 0.905 & 0.817 & -0.201 \\
04DB2 & -0.050 & -0.489 & 0.722 & 0.650 & -0.378 \\
04DB3 & 0.863 & 0.021 & 0.818 & 0.811 & -0.363 \\
\hline
\end{tabular}

According to the results, in addition to FVC2000DB2A, one can note that MQF shows a relatively stable correlation with other metrics, i.e. it exhibits an usability to variant databases. The correlation value between the proposed metric and OCL for FVC2004DB2A shows an odd value because a lot of over-inked samples are contained in this database. This problem results in some difficulties for calculating the OCL and leads to a lot of singular values. Note that the proposed metric uses only the set of minutiae location, and the fingerprint image is considered as unavailable.

\subsection{Evaluation}

According to the literature (Grother and Tabassi, 2007), the quality measure of a biometric sample is generally employed within 3 different cases: 1) including enrollment phase, 2) verification task and 3) identification. Enrollment is known as a supervised task for getting relatively good quality samples. The purpose for doing so is to guarantee the performance or reduce the recognition error as much as possible, i.e. the typical quality control operation. However, if the purpose is to validate a quality metric without considering the testing type (i.e. algorithm testing, scenario testing and so on.), the variation of the quality of enrollment sample would generate distinctive impacts on matching performance and the result is repeatable in the experiments. In this case, to estimate the validity of the MQF, an enrollment phase evaluation approach is applied to the quality metric (Yao et al., 2014).

We computed the EER values of all the trial databases by choosing the best quality samples as the enrollments (in terms of quality value generated by MQF and NFIQ). A valid quality metric for choosing the enrollment samples should reduce error rates. Two graphs with the logarithmic curves and the EER values of two different cases are given in figure 5 to illustrate the enrollment selection strategy.

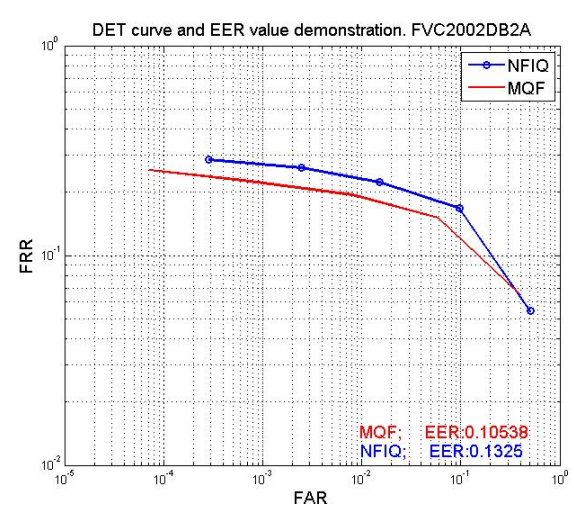

(a) FVC2002DB2A

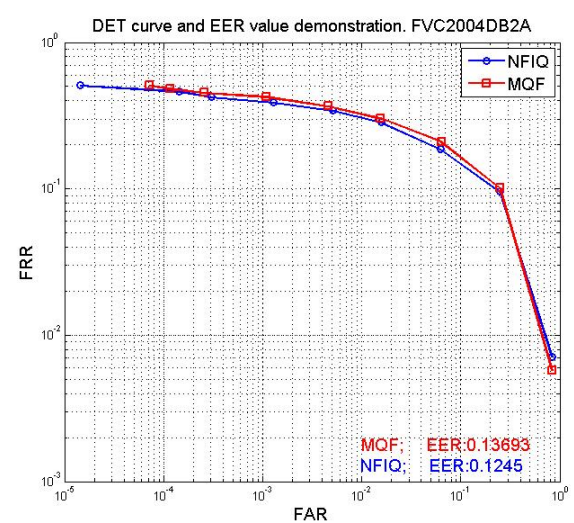

(b) FVC2004DB2A

Figure 5: The DET curves and global level EER values obtained by using the evaluation approach. $5 \mathrm{a}$ is FVC2002DB2A; 5b is FVC2004DB2A.

In figure 5, the EER values of the two datasets based on NFIQ are $13.2 \%$ (02DB2A) and $12.4 \%$ (04DB2A), while the values corresponding to MQF are: $10.5 \%$ (02DB2A), and $13.6 \%(04 \mathrm{DB} 2 \mathrm{~A})$, respectively.

Table 4: The average EER values based-on enrollment selection.

\begin{tabular}{|c|c|c|c|}
\hline $\mathrm{QM}^{\mathrm{DB}}$ & 00DB2A & 04DB1A & 04DB3A \\
\hline MQF & $5.05 \%$ & $15.03 \%$ & $6.96 \%$ \\
\hline NFIQ & $4.98 \%$ & $15.33 \%$ & $7.48 \%$ \\
\hline
\end{tabular}

According to this result, the performance of MQF is validated with the property that a good quality index reduces the matching error. However, a further validation needs to be acquired for making a conclusion that the global EER value is a statistically objective measurement of the performance. To do so, we calculate a confidence interval (CI) (Giot et al., 
2013) at 95\% level for the global EER values based on different quality metrics. In biometrics, the CI of two different measurements are able to indicate the statistical difference between them if their confidence intervals do not overlap each other. The CI values of the EER for each quality metric is calculated via a set of randomly selected intra-class and inter-class score samples after enrollment selection. The size of each kind of randomly selected matching scores is 500, and hence the $95 \% \mathrm{CI}$ is obtained with 1000 samples of the EER values, i.e. this random selection has been performed for 1000 iterations to calculate EER samples. Table 4 provides the average EER values of the other 3 databases.

Table 5: The $95 \%$ confidence interval of the EERs.

\begin{tabular}{|c|c|c|}
\hline $\mathrm{DB} \quad \mathrm{QM}$ & MQF & NFIQ \\
\hline 00DB2A (CI) & {$\left[\begin{array}{lll}0.0498 & 0.0510\end{array}\right]$} & {$\left[\begin{array}{lll}0.0492 & 0.0502\end{array}\right]$} \\
\hline 02DB2A (CI) & {$\left[\begin{array}{lll}0.1101 & 0.1118\end{array}\right]$} & {$\left[\begin{array}{lll}0.1323 & 0.1337\end{array}\right]$} \\
\hline 04DB1A (CI) & [0.1493 0.1509$]$ & {$\left[\begin{array}{lll}0.1531 & 0.1548\end{array}\right]$} \\
\hline 04DB2A (CI) & [0.1509 0.1535$]$ & {$\left[\begin{array}{lll}0.1318 & 0.1340\end{array}\right]$} \\
\hline 04DB3A (CI) & {$\left[\begin{array}{lll}0.0683 & 0.0694\end{array}\right]$} & {$\left[\begin{array}{lll}0.0742 & 0.0754\end{array}\right]$} \\
\hline
\end{tabular}

In table 5, the CI of the EER values based on the two quality metrics statistically illustrates the validity of the proposed quality metric. The overlap happened to FVC2000DB2A, for the EER values obtained from this database is nearly the same. Moreover, we simply choose several images to illustrate the differences between the two quality metrics and their drawbacks. For instance, the MQF values of the samples given in figure 6 are $(2.94,38.61,38.46,64.11,61.2)$, and their NFIQ values are all level 2 . According to sample (a), one can find that it contains only a partial of the fingerprint image which is not suitable for matching. In addition, the samples with MQF values under 40 are not as good as a level 2 sample in this example. The samples (b) and (c) would result in spurious minutiae. On the other hand, an obvious shortage of MQF is the area measurement which would generate outliers in many cases, such as the samples illustrated by figure 6 (d) and (e).

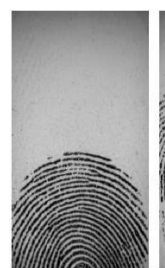

(a)

MQF: 2.94

NFIQ: 2

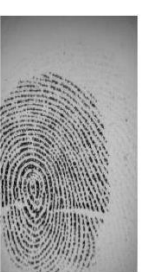

(b) 38.61 2
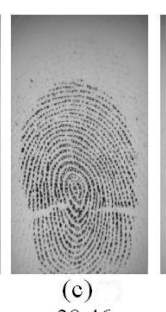

38.46

2

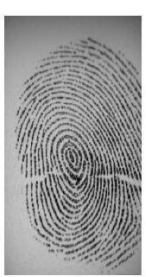

(d) 64. 11

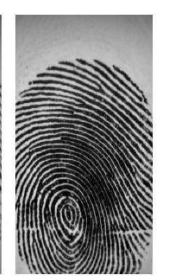

(e) 61.2
Figure 6: Illustration of the different between MQF and NFIQ.
The experiments are implemented via Matlab 7.12. The computation takes approximately 0.423 seconds per sample according to a calculation of 1600 samples when the input is image, and 0.122 seconds for template inputs.

\section{CONCLUSION}

This study mainly focuses on estimating fingerprint quality simply with a minutiae template. By investigating the relation between image-based quality criteria and the structure of minutiae template, we calculate the area of minutiae region via a convex hull and the Delaunay triangulation. The uniqueness of Delaunay triangulation provides a possibility that some unreasonable minutiae could be further eliminated from template, and the particular rules for calculating convex hull enables estimating a relatively minimum area of the detected minutiae. Accordingly, we define a simple yet efficient quality metric for fingerprint and minutiae template. In another aspect, the quality metric could be affected by some bad samples with large minutiae region. The lack of image information makes this problem inevitable. Correspondingly, we analyzed the proposed quality metric via Pearson correlation coefficients and evaluated its performance with an enrollment phase approach. Therefore, it is reasonable to conclude that the proposed quality metric is an effective measurement for assessing the quality of a various of fingerprint samples when their associated templates are available only.

As fingerprint quality metric is generally defined with multiple features, this quality metric therefore could be a candidate for those composite metrics. The future work of this study tends to concentrate on combining this quality metric with some others extracted from minutiae template to obtain better performance. 


\section{REFERENCES}

Alonso-Fernandez, F., Fierrez, J., Ortega-Garcia, J., Gonzalez-Rodriguez, J., Fronthaler, H., Kollreider, K., and Bigun, J. (2007). A comparative study of fingerprint image-quality estimation methods. Information Forensics and Security, IEEE Transactions on, 2(4):734-743.

Andrew, A. M. (1979). Another efficient algorithm for convex hulls in two dimensions. Information Processing Letters, 9(5):216-219.

Aufmann, R., Barker, V., and Nation, R. (2007). College Trigonometry. Cengage Learning.

Bolle, R. M., Pankanti, S. U., and Yao, Y.-S. (1999). System and method for determining the quality of fingerprint images. US Patent 5,963,656.

Chen, T., Jiang, X., and Yau, W. (2004). Fingerprint image quality analysis. In Image Processing, 2004. ICIP '04. 2004 International Conference on, volume 2, pages 1253-1256 Vol.2.

Delaunay, B. (1934). Sur la sphere vide. Izv. Akad. Nauk SSSR, Otdelenie Matematicheskii i Estestvennyka Nauk, 7(793-800):1-2.

Feng, J. and Jain, A. K. (2011). Fingerprint reconstruction: from minutiae to phase. Pattern Analysis and Machine Intelligence, IEEE Transactions on, 33(2):209-223.

Giot, R., El-Abed, M., and Rosenberger, C. (2013). Fast computation of the performance evaluation of biometric systems: Application to multibiometrics. Future Gener. Comput. Syst., 29(3):788-799.

Grother, P. and Tabassi, E. (2007). Performance of biometric quality measures. Pattern Analysis and Machine Intelligence, IEEE Transactions on, 29(4):531-543.

Lee, B., Moon, J., and Kim, H. (2005). A novel measure of fingerprint image quality using the Fourier spectrum. In Jain, A. K. and Ratha, N. K., editors, Society of Photo-Optical Instrumentation Engineers (SPIE) Conference Series, volume 5779 of Society of PhotoOptical Instrumentation Engineers (SPIE) Conference Series, pages 105-112.

Lim, E., Jiang, X., and Yau, W. (2002). Fingerprint quality and validity analysis. In Image Processing. 2002. Proceedings. 2002 International Conference on, volume 1, pages I-469-I-472 vol.1.

Maio, D., Maltoni, D., Cappelli, R., Wayman, J. L., and J., A. K. (2004). Fvc2004: Third fingerprint verification competition. In Biometric Authentication, pages 1-7. Springer.

Maltoni, D., Maio, D., Jain, A. K., and Prabhakar, S. (2009). Handbook of fingerprint recognition. Springer.

Nanni, L. and Lumini, A. (2007). A hybrid waveletbased fingerprint matcher. Pattern Recognition, 40(11):3146-3151.

Olsen, M., Xu, H., and Busch, C. (2012). Gabor filters as candidate quality measure for NFIQ 2.0. In Biometrics (ICB), 2012 5th IAPR International Conference on, pages 158-163.

Ratha, N. K. and Bolle, R. (1999). Fingerprint image quality estimation. IBM TJ Watson Research Center.
Staff, B. S. I. (2009). Information Technology. Biometric Sample Quality. Framework. B S I Standards.

Tabassi, E., Wilson, C., and Watson, C. (2004). NIST fingerprint image quality. NIST Res. Rep. NISTIR7151.

Watson, C. I., Garris, M. D., Tabassi, E., Wilson, C. L., Mccabe, R. M., Janet, S., and Ko, K. (2007). User's guide to nist biometric image software (nbis).

Yao, Z., Charrier, C., and Rosenberger, C. (2014). Utility validation of a new fingerprint quality metric. In International Biometric Performance Conference 2014. National Insititute of Standard and Technology (NIST).

Zhao, Q., Liu, F., and Zhang, D. (2010). A comparative study on quality assessment of high resolution fingerprint images. In Image Processing (ICIP), 2010 17th IEEE International Conference on, pages 3089-3092. IEEE. 